\title{
ARRAY PROCESSING USING TIME REVERSAL: EXPERIMENTS AND PERFORMANCE
}

\author{
José M.F. Moura, Yuanwei Jin, Dan Stancil, Jian-Gang Zhu, Ahmet Cepni, Yi Jiang and Ben Henty \\ Carnegie Mellon University \\ Department of Electrical and Computer Engineering \\ Pittsburgh, PA 15213
}

\begin{abstract}
In this paper we derive the generalized likelihood ratio test (GLRT) for time reversal detection. We consider a multistatic array configuration with two antenna arrays, one for transmitting and one for receiving. We examine the time reversal GLRT performance with experimental measurements in the electromagnetic domain in a highly cluttered laboratory environment. The experiments show that time reversal provides significant performance gain over the conventional energy detector.
\end{abstract}

\section{BACKGROUND}

Time reversal (TR) is an emerging technique for detecting weak targets buried in a large number of clutters. Time reversal has received considerable attentions in the ultrasound [1] and the acoustics domains [2]. Recently, we developed in [3] the generalized likelihood ratio (GLR) detector for a pair of transmit-receive sensors. We consider in this paper the general case of transmit-receive array $\mathrm{A}$ and array $\mathrm{B}$. The array $\mathrm{A}$ has $P$ antennas $\left[A_{0}, \cdots, A_{P-1}\right]$. The array $\mathrm{B}$ has $N$ antennas $\left[B_{0}, \cdots, B_{N-1}\right]$. Motivated by signal theory rather than electromagnetic scattering theory, we use the following two channel frequency response matrices to represent the scattering characteristics of the radar target and clutter in a multi-static configuration. The $N \times P$ clutter channel frequency response matrix $\mathbf{H}_{c}\left(f_{q}\right)$, $q=0, \cdots, Q-1$, is the response of the channel when no target is present. The $(n, p)$-th entry of $\mathbf{H}_{c}\left(f_{q}\right)$ is the channel response between antenna $A_{p}$ and antenna $B_{n}$ at frequency $f_{q}$, i.e.,

$$
\left[\mathbf{H}_{c}\left(f_{q}\right)\right]_{n, p}=H_{c}\left(f_{q} ; B_{n} \leftarrow A_{p}\right), \quad \forall p, n .
$$

Similarly, we consider the $N \times P$ target channel frequency response $\mathbf{H}_{t}\left(f_{q}\right), q=0, \cdots, Q-1$, which is the difference between the channel response when a target is present and the channel response when no target is present. Again,

This work is funded by the Defence Advanced Research Project Agency through the Army Research Office under grant no. W911NF-04$1-0031$. the $(n, p)$-th entry of $\mathbf{H}_{t}\left(f_{q}\right)$ is the target channel response between antennas $A_{p}$ and $B_{n}$, i.e.,

$$
\left[\mathbf{H}_{t}\left(f_{q}\right)\right]_{n, p}=H_{t}\left(f_{q} ; B_{n} \leftarrow A_{p}\right), \quad \forall p, n .
$$

As such, $\mathbf{H}_{t}\left(f_{q}\right)$ represents all the changes to $\mathbf{H}_{c}\left(f_{q}\right)$ induced by the presence of the target, and, in particular, it includes secondary backscatter, i.e., backscatter from the clutter to the target that is then radiated back to the receiving antenna. Throughout the paper, it is assumed that the clutter channel frequency response $\mathbf{H}_{c}\left(f_{q}\right), \forall q$, can be learned and can be subtracted out (see [3] for details). It suffices to consider $\mathbf{H}_{t}\left(f_{q}\right)$ for detector development.

The general transmission strategy using time reversal can be described by the following three steps. First, we learn the scattering channel response. Second, we monitor the target channel, while the clutter channel response is subtracted from the measurements. Finally, we re-transmit the power-normalized time-reversed signal back into the scattering environment. In general, the target response is unknown. We adopt the generalized likelihood ratio (GLR) principle to derive the time reversal GLRT (TR-GLRT) detector. We test the TR-GLRT with electromagnetic data collected in a highly cluttered laboratory environment.

\section{TIME REVERSAL DATA MODEL}

We denote by $Y_{m, p, n}\left(f_{q}\right)$ the received $m$-th snapshot signal at antenna $B_{n}$ transmitted from antenna $A_{p}$ at $f_{q}$ where $m=1, \cdots, M$. Grouping all the measurements collected from the receiving antennas $B_{1}, \cdots, B_{N}$ yields an $N$ dimensional signal vector

$$
\mathbf{y}_{m, p}\left(f_{q}\right)=\left[Y_{m, p, 0}\left(f_{q}\right), \cdots, Y_{m, p, N-1}\left(f_{q}\right)\right]^{T}, \quad \forall p, m .
$$

Using the target frequency response $\mathbf{H}_{t}\left(f_{q}\right)$, the received signal vector at the array of antennas $\mathrm{B}$ transmitted from antenna $A_{p}$ is

$$
\mathbf{y}_{m, p}\left(f_{q}\right)=\mathbf{H}_{t}\left(f_{q}\right) S\left(f_{q}\right) \mathbf{e}_{p}+\mathbf{v}_{m, p}\left(f_{q}\right), \quad \forall p, m,
$$

where $\mathbf{e}_{p}$ is a column vector of zero entries except that its $p$ th element is $1 ; S\left(f_{q}\right)$ is the transmitted signal at frequency 
$f_{q}$; and $\mathbf{v}_{m, p}\left(f_{q}\right)$ is an additive noise vector. The total received signal for the $m$-th snapshot at array $\mathrm{B}$, transmitted from array $\mathrm{A}$, is

$$
\mathbf{y}_{m}\left(f_{q}\right)=\sum_{p=0}^{P-1} \mathbf{y}_{m, p}\left(f_{q}\right)=\mathbf{H}_{t}\left(f_{q}\right) \mathbf{s}_{A}\left(f_{q}\right)+\mathbf{v}_{m}\left(f_{q}\right),
$$

where the signal vector $\mathbf{s}_{A}\left(f_{q}\right)$ and the noise vector $\mathbf{v}_{m}\left(f_{q}\right)$ are

$$
\begin{aligned}
\mathbf{s}_{A}\left(f_{q}\right) & =\left[S_{1}\left(f_{q}\right), \cdots, S_{P}\left(f_{q}\right)\right]^{T} \\
\mathbf{v}_{m}\left(f_{q}\right) & =\left[V_{m, 0}\left(f_{q}\right), \cdots, V_{m, N-1}\left(f_{q}\right)\right]^{T} .
\end{aligned}
$$

We assume that $S_{1}\left(f_{q}\right)=\cdots=S_{P}\left(f_{q}\right)$. For snapshot $m$, collecting the frequency response $\mathbf{y}_{m}\left(f_{q}\right), q=0, \cdots, Q-$ 1 , in a $N Q$-dimensional vector $\mathbf{y}_{m}$ yields

$$
\mathbf{y}_{m}=\left[\mathbf{y}_{m}^{T}\left(f_{0}\right), \cdots, \mathbf{y}_{m}^{T}\left(f_{Q-1}\right)\right]^{T} .
$$

The signal vector $\mathbf{y}_{m}$ received at the array of antennas B are now time reversed, power normalized, and transmitted back into the scattering medium after subtracting out the clutter response. The $P \times 1$ received signal vector at antenna array $\mathrm{A}$ is

$$
\begin{aligned}
\mathbf{x}_{m}\left(f_{q}\right)= & k_{m} \mathbf{H}_{t}^{T}\left(f_{q}\right)\left[\mathbf{y}_{m}\left(f_{q}\right)\right]^{*}+\mathbf{w}_{m}\left(f_{q}\right), \\
= & k_{m} \mathbf{H}_{t}^{T}\left(f_{q}\right)\left[\mathbf{H}_{t}^{*}\left(f_{q}\right) \mathbf{s}_{A}^{*}\left(f_{q}\right)\right. \\
& \left.+\mathbf{v}_{m}^{*}\left(f_{q}\right)\right]+\mathbf{w}_{m}\left(f_{q}\right),
\end{aligned}
$$

where $\mathbf{w}_{m}$ is the complex Gaussian noise vector

$$
\mathbf{w}_{m}\left(f_{q}\right)=\left[W_{m, 0}\left(f_{q}\right), \cdots, W_{m, P-1}\left(f_{q}\right)\right]^{T}, \quad \forall p, m .
$$

Due to reciprocity of the channel, the target response from $\mathrm{B}$ back to $\mathrm{A}$ is $\mathbf{H}_{t}^{T}\left(f_{q}\right)$. The gain $k_{m}$ is the power normalization factor for each antenna $B_{n}$

$$
k_{m}=\sqrt{\frac{\sum_{q=0}^{Q-1}\left\|\mathbf{s}_{A}\left(f_{q}\right)\right\|^{2}}{\sum_{q=0}^{Q-1}\left\|\mathbf{y}_{m}\left(f_{q}\right)\right\|^{2}}} .
$$

We define data vectors $\mathbf{x}_{m}\left(f_{q}\right), \mathbf{v}_{m}\left(f_{q}\right)$, and $\mathbf{w}_{m}\left(f_{q}\right)$

$$
\begin{aligned}
\mathbf{x}_{m} & =\left[\mathbf{x}_{m}^{T}\left(\omega_{0}\right), \cdots, \mathbf{x}_{m}^{T}\left(\omega_{Q-1}\right)\right]^{T}, \\
\mathbf{v}_{m} & =\left[\mathbf{v}_{m}^{T}\left(\omega_{0}\right), \cdots, \mathbf{v}_{m}^{T}\left(\omega_{Q-1}\right)\right]^{T}, \\
\mathbf{w}_{m} & =\left[\mathbf{w}_{m}^{T}\left(\omega_{0}\right), \cdots, \mathbf{w}_{m}^{T}\left(\omega_{Q-1}\right)\right]^{T} .
\end{aligned}
$$

The detection problem using time reversal can now be formulated as a binary hypothesis test obtained by concatenating the measurements $\left(\mathbf{y}_{m}, \mathbf{x}_{m}\right), \forall m$. We also consider a second detection problem - the conventional detection. This will use concatenated data measurements $\left(\mathbf{y}_{m}, \mathbf{r}_{m}\right)$, where $\mathbf{y}_{m}$ is given in (7) and

$$
\begin{aligned}
\mathbf{r}_{m}\left(f_{q}\right) & =\mathbf{H}^{T}\left(f_{q}\right) \mathbf{s}_{B}\left(f_{q}\right)+\mathbf{w}_{m}\left(f_{q}\right), \\
\mathbf{r}_{m} & =\left[\mathbf{r}_{m}\left(\omega_{0}\right), \cdots, \mathbf{r}_{m}\left(\omega_{Q-1}\right)\right]^{T}, \\
\mathbf{s}_{B}\left(f_{q}\right) & =\left[S_{1}\left(f_{q}\right), \cdots, S_{N}\left(f_{q}\right)\right]^{T} .
\end{aligned}
$$

Time reversal detection uses $\left(\mathbf{y}_{m}, \mathbf{x}_{m}\right)$ and conventional detection uses $\left(\mathbf{y}_{m}, \mathbf{r}_{m}\right)$. This ensures that, for benchmarking purposes, the SNR will be the same in both problems.

\section{TIME REVERSAL GLRT}

We collect the data in the following $Q(P+N)$ dimensional vector $\mathbf{z}_{m}=\left[\begin{array}{ll}\left(\mathbf{y}_{m}^{*}\right)^{T} & \mathbf{x}_{m}^{T}\end{array}\right]^{T}$. The time reversal binary hypothesis test is then given by

$$
\begin{aligned}
& \mathbb{H}_{1}: \quad \mathbf{z}_{m}=\left[\begin{array}{c}
\mathbf{y}_{t}^{*} \\
\mathbf{x}_{t}
\end{array}\right]+\left[\begin{array}{c}
\mathbf{v}_{m}^{*} \\
\mathbf{w}_{m} \\
\mathbf{v}_{m}^{*} \\
\mathbf{w}_{m}
\end{array}\right] \\
& \mathbb{H}_{0}: \quad \mathbf{z}_{m}=
\end{aligned}
$$

where the target signal components $\mathbf{y}_{t}=\mathbf{H}_{t}\left(f_{q}\right) \mathbf{s}_{A}\left(f_{q}\right)$ and $\mathbf{x}_{t}=k_{m} \mathbf{H}_{t}^{T}\left(f_{q}\right) \mathbf{H}_{t}^{*}\left(f_{q}\right) \mathbf{s}_{A}^{*}\left(f_{q}\right)$. The conventional detection problem is similarly defined with proper modifications. For the hypothesis test (15), the $m$-th snapshot under the alternative hypothesis is a complex Gaussian vector

$$
\begin{aligned}
& \mathbf{z}_{m} \sim \mathcal{C N}\left(\left[\begin{array}{c}
\mathbf{H}^{*}\left(f_{q}\right) \mathbf{s}_{A}^{*}\left(f_{q}\right) \\
k_{m} \mathbf{H}_{t}^{T}\left(f_{q}\right) \mathbf{H}_{t}^{*}\left(f_{q}\right) \mathbf{s}_{A}^{*}
\end{array}\right], \boldsymbol{\Omega}(q)\right), \\
& \boldsymbol{\Omega}(q)=\left[\begin{array}{ll}
\sigma_{v}^{2} \mathbf{I}_{N} & k_{m} \mathbf{H}_{t}^{*}\left(f_{q}\right) \sigma_{v}^{2} \\
k_{m} \mathbf{H}_{t}^{T}\left(f_{q}\right) \sigma_{v}^{2} & k_{m}^{2} \mathbf{H}_{t}^{T}\left(f_{q}\right) \mathbf{H}_{t}^{*}\left(f_{q}\right) \sigma_{v}^{2}+\sigma_{w}^{2} \mathbf{I}_{P}
\end{array}\right] .
\end{aligned}
$$

Using the matrix inversion lemma, [4], and the block matrix inversion lemma, [4], we obtain

$\boldsymbol{\Omega}^{-1}(q)=\left[\begin{array}{ll}\frac{1}{\sigma_{v}^{2}} \mathbf{I}_{N}+\frac{k_{m}^{2}}{\sigma_{w}^{2}} \mathbf{H}_{t}^{*}\left(f_{q}\right) \mathbf{H}_{t}^{T}\left(f_{q}\right) & -\frac{k_{m}}{\sigma_{w}^{2}} \mathbf{H}_{t}^{*}\left(f_{q}\right) \\ -\frac{k_{m}}{\sigma_{w}^{2}} \mathbf{H}_{t}^{T}\left(f_{q}\right) & \frac{1}{\sigma_{w}^{2}} \mathbf{I}_{P}\end{array}\right]$.

Applying the matrix determinant identity, [4],

$$
\operatorname{det}\left(\left[\begin{array}{ll}
\mathbf{A} & \mathbf{B} \\
\mathbf{C} & \mathbf{D}
\end{array}\right]\right)=\operatorname{det}(\mathbf{A}) \operatorname{det}\left(\mathbf{D}-\mathbf{C A}^{-1} \mathbf{B}\right)
$$

to $\boldsymbol{\Omega}(q)$ yields

$$
|\boldsymbol{\Omega}(q)|=\left|\sigma_{v}^{2} \mathbf{I}_{N}\right|\left|\sigma_{w}^{2} \mathbf{I}_{P}\right|=\left(\sigma_{v}^{2}\right)^{N}\left(\sigma_{w}^{2}\right)^{P} .
$$

For snapshot $m=1, \cdots, M$, we define

$$
\begin{aligned}
& \overline{\mathbf{y}}_{m}\left(f_{q}\right)=\mathbf{y}_{m}^{*}\left(f_{q}\right)-\mathbf{H}_{t}^{*}\left(f_{q}\right) \mathbf{s}^{*}\left(f_{q}\right) \\
& \overline{\mathbf{x}}_{m}\left(f_{q}\right)=\mathbf{x}_{m}\left(f_{q}\right)-k_{m} \mathbf{H}_{t}^{T}\left(f_{q}\right) \mathbf{H}_{t}^{*}\left(f_{q}\right) \mathbf{s}^{*}\left(f_{q}\right) .
\end{aligned}
$$

To derive the TR-GLRT, we need the probability density functions (pdfs) $p_{1}(\mathbf{z})$ under $\mathbb{H}_{1}$ and $p_{0}(\mathbf{z})$ under $\mathbb{H}_{0}$. We condition the pdfs on the power normalization factors $k_{m}$. 
We then obtain

$$
\begin{aligned}
& p_{1}(\mathbf{z})=\prod_{m=1}^{M} \prod_{q=0}^{Q-1} \frac{1}{\pi|\boldsymbol{\Omega}(q)|} \exp \left\{-\left[\begin{array}{c}
\overline{\mathbf{y}}_{m}\left(f_{q}\right) \\
\overline{\mathbf{x}}_{m}\left(f_{q}\right)
\end{array}\right]^{H}\right. \\
& \left.\boldsymbol{\Omega}^{-1}(q)\left[\begin{array}{l}
\overline{\mathbf{y}}_{m}^{*}\left(f_{q}\right) \\
\overline{\mathbf{x}}_{m}\left(f_{q}\right)
\end{array}\right]\right\} \\
& =\prod_{m=1}^{M} \prod_{q=0}^{Q-1} \frac{1}{\pi\left(\sigma_{w}^{2}\right)^{P}\left(\sigma_{v}^{2}\right)^{N}} \exp \left\{-\overline{\mathbf{y}}_{m}^{H}\left(f_{q}\right)\right. \\
& {\left[\frac{1}{\sigma_{v}^{2}} \mathbf{I}_{N}+\frac{k_{m}^{2}}{\sigma_{w}^{2}} \mathbf{H}_{t}^{*}\left(f_{q}\right) \mathbf{H}_{t}^{T}\left(f_{q}\right)\right] \overline{\mathbf{y}}_{m}^{*}\left(f_{q}\right)} \\
& \left.+2 \Re\left\{\frac{k_{m}}{\sigma_{w}^{2}} \overline{\mathbf{x}}_{m}^{H}\left(f_{q}\right) \mathbf{H}_{t}^{T}\left(f_{q}\right) \overline{\mathbf{y}}_{m}^{*}\left(f_{q}\right)\right\} \frac{1}{\sigma_{w}^{2}}\left\|\overline{\mathbf{x}}_{m}\left(f_{q}\right)\right\|^{2}\right\} \\
& =\prod_{m=1}^{M} \prod_{q=0}^{Q-1} \frac{1}{\pi\left(\sigma_{w}^{2}\right)^{P}\left(\sigma_{v}^{2}\right)^{N}} \exp \left\{-\frac{\left\|\mathbf{x}_{m}\left(f_{q}\right)\right\|^{2}}{\sigma_{w}^{2}}\right. \\
& -\frac{\left\|\mathbf{y}_{m}\left(f_{q}\right)\right\|^{2}}{\sigma_{v}^{2}}+2 \Re\left\{\frac{k_{m}}{\sigma_{w}^{2}} \mathbf{y}_{m}^{T}\left(f_{q}\right) \mathbf{H}_{t}^{*}\left(f_{q}\right) \mathbf{x}_{m}\left(f_{q}\right)\right. \\
& \left.+\frac{1}{\sigma_{v}^{2}} \mathbf{y}_{m}^{T}\left(f_{q}\right) \mathbf{H}_{t}^{*}\left(f_{q}\right) \mathbf{s}^{*}\left(f_{q}\right)\right\} \\
& \left.-\frac{1}{\sigma_{v}^{2}}\left\|\mathbf{H}_{t}^{*}\left(f_{q}\right) \mathbf{s}^{*}\left(f_{q}\right)\right\|^{2}-\frac{k_{m}^{2}}{\sigma_{w}^{2}}\left\|\mathbf{H}_{t}^{T}\left(f_{q}\right) \mathbf{y}_{m}^{*}\left(f_{q}\right)\right\|^{2}\right\} \\
& p_{0}(\mathbf{z})=\prod_{m=1}^{M} \prod_{q=0}^{Q-1} \frac{1}{\pi\left(\sigma_{w}^{2}\right)^{P}\left(\sigma_{v}^{2}\right)^{N}} \exp \left\{-\frac{\left\|\mathbf{x}_{m}\left(f_{q}\right)\right\|^{2}}{\sigma_{w}^{2}}\right\} \\
& \exp \left\{-\frac{\left\|\mathbf{y}_{m}\left(f_{q}\right)\right\|^{2}}{\sigma_{v}^{2}}\right\} \text {. }
\end{aligned}
$$

We now derive the TR-GLRT. Taking the logarithm of the ratio of the two pdfs (19) and (20) evaluated at the maximum likelihood estimate of $\mathbf{H}_{t}$ yields the test statistic

$$
\begin{aligned}
& \ell_{\mathrm{TR}-\mathrm{GLRT}}(\mathbf{z})= \ln p_{1}(\mathbf{z})-\ln p_{0}(\mathbf{z}) \\
&= \sum_{m=1}^{M} \sum_{q=0}^{Q-1}\left\{2 \Re\left\{\frac{k_{m}}{\sigma_{w}^{2}} \mathbf{y}_{m}^{T}\left(f_{q}\right) \widehat{\mathbf{H}}_{t}^{*}\left(f_{q}\right) \mathbf{x}_{m}\left(f_{q}\right)\right\}\right. \\
&+ 2 \Re\left\{\frac{1}{\sigma_{v}^{2}} \mathbf{y}_{m}^{T}\left(f_{q}\right) \widehat{\mathbf{H}}_{t}^{*}\left(f_{q}\right) \mathbf{s}^{*}\left(f_{q}\right)\right\} \\
&\left.-\frac{1}{\sigma_{v}^{2}}\left\|\widehat{\mathbf{H}}_{t}^{*}\left(f_{q}\right) \mathbf{s}^{*}\left(f_{q}\right)\right\|^{2}-\frac{k_{m}^{2}}{\sigma_{w}^{2}}\left\|\widehat{\mathbf{H}}_{t}^{T}\left(f_{q}\right) \mathbf{y}_{m}^{*}\left(f_{q}\right)\right\|^{2}\right\} \\
&=\frac{1}{\sigma_{w}^{2} \sigma_{v}^{2}} \sum_{q=0}^{Q-1}\left\{2 \Re\left\{\operatorname{Tr}\left[\widehat{\mathbf{H}}_{t}^{*}\left(f_{q}\right) \mathbf{A}\left(f_{q}\right)\right]\right\}\right. \\
&-2 \Re\left\{\operatorname{Tr}\left[\widehat{\mathbf{H}}_{t}^{T}\left(f_{q}\right) \widehat{\mathbf{H}}_{t}^{*}\left(f_{q}\right) \mathbf{C}\left(f_{q}\right)\right]\right. \\
&\left.-\operatorname{Tr}\left[\widehat{\mathbf{H}}_{t}^{*}\left(f_{q}\right) \widehat{\mathbf{H}}_{t}^{T}\left(f_{q}\right) \mathbf{D}\left(f_{q}\right)\right]\right\}
\end{aligned}
$$

where

$$
\mathbf{A}\left(f_{q}\right)=\sum_{m=1}^{M}\left[k_{m} \sigma_{v}^{2} \mathbf{x}_{m}\left(f_{q}\right) \mathbf{y}_{m}^{T}\left(f_{q}\right)+\sigma_{w}^{2} \mathbf{s}^{*}\left(f_{q}\right) \mathbf{y}_{m}^{T}\left(f_{q}\right)\right]
$$

$$
\begin{aligned}
\mathbf{C}\left(f_{q}\right) & =M \sigma_{w}^{2} \mathbf{s}^{*}\left(f_{q}\right) \mathbf{s}^{T}\left(f_{q}\right), \\
\mathbf{D}\left(f_{q}\right) & =\sum_{m=1}^{M} k_{m}^{2} \sigma_{v}^{2} \mathbf{y}_{m}^{*}\left(f_{q}\right) \mathbf{y}_{m}^{T}\left(f_{q}\right),
\end{aligned}
$$

and $\widehat{\mathbf{H}}_{t}\left(f_{q}\right)$ is the maximum likelihood estimate of $\mathbf{H}_{t}\left(f_{q}\right)$ under $\mathbb{H}_{1}$ to be determined below. This is not a linear test statistic, which is to be expected given that the channel is unknown.

Next, we derive the maximum likelihood estimate of $\mathbf{H}_{t}$ under $\mathbb{H}_{1}$. Our derivation neglects the dependency of the power normalization factors $k_{m}$ on the target channel response and so is only an approximation to the true ML estimate. Taking the partial derivative of $\ln p_{1}(\mathbf{z})$ with respect to $\mathbf{H}_{t}^{*}\left(f_{q}\right)$, and ignoring the constant terms, yields

$$
\begin{gathered}
\frac{\partial\left[\ln p_{1}(\mathbf{z})\right]}{\partial \mathbf{H}_{t}^{*}\left(f_{q}\right)}=\frac{\partial}{\partial \mathbf{H}_{t}^{*}\left(f_{q}\right)} \sum_{q=0}^{Q-1}\left\{-\sum_{m=1}^{M} \frac{\left\|\mathbf{x}_{m}\left(f_{q}\right)\right\|^{2}}{\sigma_{w}^{2}}\right. \\
-\sum_{m=1}^{M} \frac{\left\|\mathbf{y}_{m}\left(f_{q}\right)\right\|^{2}}{\sigma_{v}^{2}}+2 \mathfrak{R}\left\{\operatorname{Tr}\left[\mathbf{H}_{t}^{*}\left(f_{q}\right) \mathbf{A}\left(f_{q}\right)\right]\right\} \\
-\operatorname{Tr}\left[\mathbf{H}_{t}^{T}\left(f_{q}\right) \mathbf{H}_{t}^{*}\left(f_{q}\right) \mathbf{C}\left(f_{q}\right)\right] \\
\left.-\operatorname{Tr}\left[\mathbf{H}_{t}^{*}\left(f_{q}\right) \mathbf{H}_{t}^{T}\left(f_{q}\right) \mathbf{D}\left(f_{q}\right)\right]\right\} .
\end{gathered}
$$

Recalling the rule of treating the complex variable $v$ and its conjugate $v^{*}$ as two distinct quantities, i.e., $\frac{\partial|v|^{2}}{\partial v^{*}}=v$, [5], we obtain the following expression that involves taking the derivative of a trace with respect to complex numbers,

$$
\frac{\partial\left[\ln p_{1}(\mathbf{z})\right]}{\partial \mathbf{H}_{t}^{*}\left(f_{q}\right)}=-\mathbf{C}\left(f_{q}\right) \mathbf{H}_{t}^{T}\left(f_{q}\right)-\mathbf{H}_{t}^{T}\left(f_{q}\right) \mathbf{D}\left(f_{q}\right)+\mathbf{A}\left(f_{q}\right) .
$$

Or equivalently the following well known Lyapunov equation (or Sylvester matrix equation), [6],

$$
\mathbf{H}_{t}\left(f_{q}\right) \mathbf{C}^{T}\left(f_{q}\right)+\mathbf{D}^{T}\left(f_{q}\right) \mathbf{H}_{t}\left(f_{q}\right)=\mathbf{A}^{T}\left(f_{q}\right) .
$$

A straightforward numerical approach to the above equation forms the single vector equation using Kronecker products as follows

$$
\begin{aligned}
\operatorname{vec}\left\{\mathbf{A}^{T}\left(f_{q}\right)\right\} & =\mathbf{G}\left(f_{q}\right) \operatorname{vec}\left\{\mathbf{H}_{t}\left(f_{q}\right)\right\}, \\
\mathbf{G}\left(f_{q}\right) & =\mathbf{C}\left(f_{q}\right) \otimes \mathbf{I}_{N}+\mathbf{I}_{P} \otimes \mathbf{D}^{T}\left(f_{q}\right),
\end{aligned}
$$

which leads to

$$
\operatorname{vec}\left\{\mathbf{H}_{t}\left(f_{q}\right)\right\}=\left[\mathbf{G}\left(f_{q}\right)\right]^{-1} \operatorname{vec}\left\{\mathbf{A}^{T}\left(f_{q}\right)\right\} .
$$

The approximate ML estimate $\widehat{\mathbf{H}}_{t}\left(f_{q}\right)$ is obtained by undoing the vectorization of vec $\left\{\mathbf{H}_{t}\left(f_{q}\right)\right\}$ given in Eqn. (27). Caution should be taken when completing this ML estimate from (27) since it is often slow and ill-conditioned. Other standard solution methods for (26) are the Bartels-Stewart method, [7], and the Hessenberg-Schur method, [8]. 


\section{EXPERIMENTS AND SIMULATIONS}

We measured the electromagnetic channel response with 30 scatterers in a laboratory environment. The experiments were repeated with the same 30 scatterers plus one additional target. The signal $S\left(f_{q}\right)$ is a rectangular frequency pulse in the $4-6 \mathrm{GHz}$ range. We choose $Q=51$ equally spaced frequencies. Two horn antennas are used to synthesize two uniform linear arrays, each with $P=N=10$ antenna elements. There are a total of $B_{n} \leftarrow A_{p} 10 \times 10$ channels recorded. The different array configurations can be set by choosing among 10 antenna positions from each transmit/receive array. The scattering environment is created by placing a mix of copper rods and dielectric pipes in a wood platform in front of an absorber wall. An Agilent 89610 vector signal analyzer is used to capture the in-phase (I channel) and quadrature (Q channel) streams of the response. After the channel responses with and without target are recorded, artificial noise is inserted in the measurements with SNR $=\sum_{q=0}^{Q-1}\left\|\mathbf{H}_{t}\left(\omega_{q}\right) \mathbf{s}_{A}\left(\omega_{q}\right)\right\|^{2} /\left(N Q \sigma_{v}^{2}\right)$. Fig. 2 depicts the ROC curves of the TR-GLRT versus conventional energy detection. As an example, the array configuration is $A=[1,3,7]$ and $B=[3,6]$. The number of data snapshots used for detection is $M=2$. The false alarm rate $P_{f a}=0.01$. For probability of detection $P_{D}=0.8$, the TR-GLRT has a detection gain of $1.8 \mathrm{~dB}$ over the conventional energy detector (ED).

\section{CONCLUSION}

We derived the time reversal GLRT detector using antenna arrays and compared its performance with the conventional energy detector. We developed a closed form expression for the approximate ML estimate of the target channel response. The detector is tested using electromagnetic data collected in a rich scattering laboratory environment with 30 scatterers. The ROC curves demonstrate the superiority of the TR-GLRT over the conventional detectors.

\section{REFERENCES}

[1] M. Fink, "Time reversed acoustics," Physics Today, vol. 50, no. 3, pp. 34-40, 1997.

[2] W. A. Kuperman, W. S. Hodgkiss, and H. C. Song, "Phase conjugation in the ocean: Experimental demonstration of an acoustic time-reversal mirror," J. Acoustic Society of America, vol. 103, no. 1, pp. 25-40, January 1998.

[3] J. M. F. Moura and Y. Jin, "Detection by time reversal: single antenna," July 2005, submitted for publication.

[4] R. A. Horn and C. R. Johnson, Matrix Analysis. New York: Cambridge University Press, 1985.

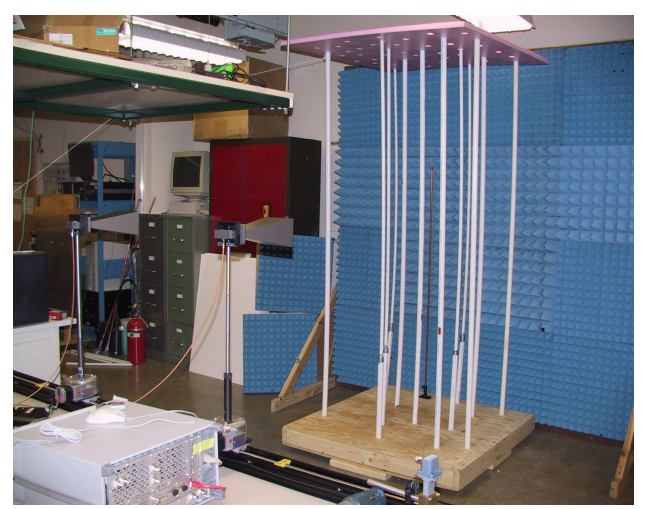

Fig. 1. Experimental setup for time reversal detection using antenna arrays. Two horn antennas are used to synthesize two linear arrays.

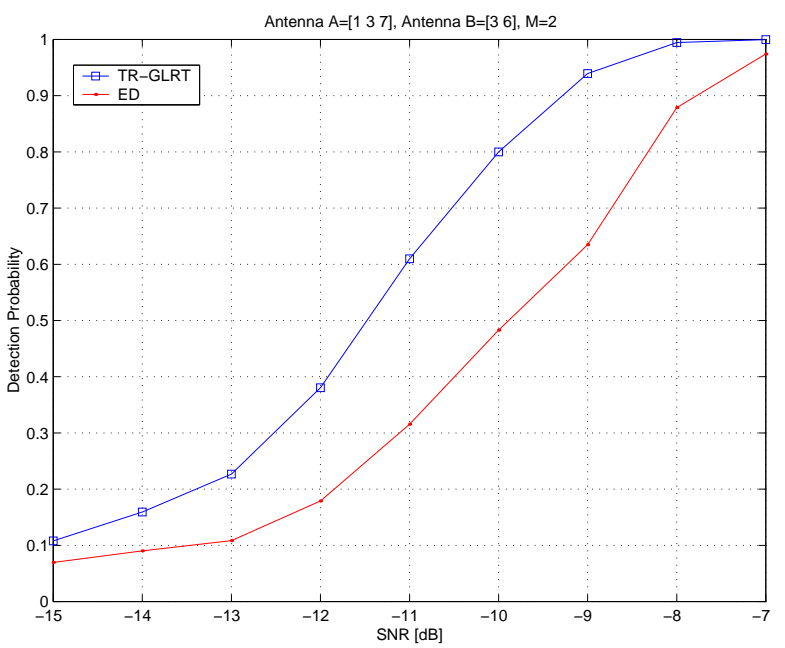

Fig. 2. ROC: TR-GLRT and conventional energy detector (ED); 30 scatterers; $M=2$. SNR gain at $P_{d}=0.8$ is 1.8 $\mathrm{dB}$.

[5] M. H. Hayes, Statistical Digital Signal Processing and Modeling. New York, NY: John Wiley \& Sons, Inc., 1996.

[6] G. Golub and C. V. Loan, Matrix computations. Baltimore, MD: The Johns Hopkins University Press, 1989.

[7] R. H. Bartels and G. W. Stewart, "A solution of the equation $\mathrm{AX}+\mathrm{XB}=\mathrm{C}$," Communications of the $A C M$, vol. 15 , no. 9 , pp. 820-826, 1972.

[8] G. H. Golub, S. Nash, and C. V. Loan, "A HessenbergSchur method for the problem $\mathrm{AX}+\mathrm{XB}=\mathrm{C}$," IEEE Transactions on Automatic Control, vol. AC-24, no. 6, pp. 909-913, December 1979. 